\title{
Arte reabilitação em mulheres amputadas utilizando o mito de Pandora como recurso facilitador de autoestima e qualidade de vida
}

\section{Art rehabilitation in amputee women with Pandora's myth as a self-esteem and quality of life facilitator resource}

Flavia Rodrigues de Souza Scorachio ${ }^{1}$, Teresa Kam Teng ${ }^{1}$, Márcia Gallo De Conti², Tania Cristina Freire², Sheila Jean McNeill Ingham ${ }^{3}$

\section{RESUMO}

A amputação é um evento traumático que repercute intensamente na vida da pessoa acometida. A dificuldade em lidar com a nova realidade pode contribuir negativamente para a autoestima e reabilitação do indivíduo, afetando a sua qualidade de vida. A Arteterapia por meio dos recursos expressivos pode ser um canal facilitador e promotor de aspectos resilientes para a superação do trauma. Objetivo: Averiguar a influência da Arteterapia na autoestima e qualidade de vida em mulheres amputadas. Grupo formado por 8 mulheres amputadas, entre 35 a 65 anos. Método: Divididos em 2 grupos (4 indivíduos no grupo de intervenção e 4 indivíduos no grupo controle). Instrumentos de avaliação: WHOQOL- Bref (World Health Organization Quality of Life), Escala de Autoestima Rosemberg (EAR), o Desenho da Figura Humana e Relatos das Participantes. As atividades foram desenvolvidas com base no mito de Pandora, em 11 oficinas arteterapêuticas com 1 hora de duração, uma vez por semana no setor de Arte-Reabilitação, AACD - Ibirapuera, São Paulo. Resultados: Estatisticamente não foram observadas diferenças significantes entre os momentos inicial e final para os domínios de Whoqol Bref e EAR, em ambos os grupos; porém, o grupo intervenção apresenta um movimento de melhora na autoestima, especialmente no quesito autodepreciação. Qualitativamente foram observadas através do discurso das participantes melhorias de autoestima e possibilidade de melhoria na qualidade de vida das participantes. Conclusão: A arteterapia, junto com a equipe interdisciplinar, pode contribuir positivamente para o processo de reabilitação em mulheres amputadas ajudando a promover a autoestima e qualidade de vida.

Palavras-chave: Amputação, Mulheres, Terapia pela Arte, Reabilitação, Qualidade de Vida

\begin{abstract}
${ }^{1}$ Aprimoranda, Setor de Arte-Reabilitação da Associação de Assistência à Criança Deficiente-AACD.

${ }^{2}$ Arteterapeuta, Associação de Assistência à Criança Deficiente - AACD.

${ }^{3}$ Médica Fisiatra, Associação de Assistência à Criança Deficiente - AACD, Setor de Fisiatria da Universidade Federal de São Paulo - UNIFESP.
\end{abstract}

\section{Correspondência}

Tania Cristina Freire

E-mail: taniafreirearteterapeuta@gmail.com

Recebido: 25 Abril 2018.

Aceito: 06 Junho 2018.

\section{Como citar}

Scorachio FRS, Teng TK, De Conti MG, Freire TC, Ingham SJM. Arte reabilitação em mulheres amputadas utilizando o mito de Pandora como recurso facilitador de autoestima e qualidade de vida. Acta Fisiatr. 2018;25(1):12-18.

\begin{abstract}
Amputation is a traumatic event that has serious repercussions on the amputee's life. The difficulty in dealing with the new reality can contribute negatively to self-esteem and rehabilitation, affecting the patient's quality of life. The Art therapy, through its expressive resources, can be a facilitator and provide resilience to overcoming the trauma. Objective: The objective of this study is to investigate the influence of art therapy in self-esteem and quality of life in amputee women. Methods: A group of 8 amputees, aged 35-65 years were divided into 2 groups (4 in the experimental group and 4 in the control group). They were evaluated with the WHOQoL-Bref (World Health Organization Quality of Life), Rosenberg Self-Esteem Scale (RSE), Human Figure Drawing test and the participants' own Reports. The activities, based on the Pandora myth, were developed in 11 art therapy workshops sessions of one hour that were carried out once a week in the Art-Rehabilitation sector off AACD - Ibirapuera, São Paulo. Results: There was no statistically significant differences observed between the initial and final assessments of quality of life (WHOQoL-Bref) and RSE domains of both groups, however, the experimental group evidenced a tendency towards the improvement of self-esteem, especially in regards of self-depreciation. Qualitatively, improvements of self-esteem and the possibility of improving the participants' quality of life were also observed. Conclusion: Art Therapy, combined with interdisciplinary care, can contribute positively to the rehabilitation process of amputee women, by helping to promote better self-esteem and quality of life.
\end{abstract}

Keywords: Amputation, Women, Art Therapy, Rehabilitation, Quality of Life 


\section{INTRODUÇÃO}

A amputação refere-se à ausência total ou parte de um membro, associadas às causas congênitas ou adquiridas. Estudos mostram que as causas mais frequentes de amputação de membro inferior são os acidentes, as doenças vasculares e o diabetes mellitus. Conforme Ramos et al. ${ }^{1}$ os motivos não traumáticos respondem por volta de $75 \%$ das amputações. A doença vascular periférica é a principal causa de amputações em pacientes não diabéticos e é responsável por cerca de $50 \%$ das amputações em pacientes diabéticos. Em segundo lugar aparecem as etiologias traumáticas, devidas aos acidentes de trânsito ou de trabalho, e em menor incidência, os tumores e as infecções. ${ }^{1}$

A amputação de um membro é um acontecimento muito difícil, delicado e quase sempre imprevisível. A perda de uma parte do corpo gera um grande impacto na vida com uma série de alterações biopsicosocial que interferem nos papéis exercidos pelo indivíduo nos âmbitos: pessoal, social, familiar e profissional. Os sentimentos tornam-se ambíguos e confusos, por um lado a razão entende a cirurgia como necessária, que evita um mal maior; mas por outro lado, há o fator emocional que resiste em aceitar a perda dificultando a recuperação do indivíduo. As emoções mais frequentes que surgem após a amputação são: o sentimento de impotência, o estranhamento de si mesmo, a baixa autoestima, a perda da identidade, a angústia, a falta de sentido e de motivação, a indefinição em relação ao futuro, às capacidades e às limitações que estão sendo vividas. , $3,4^{2,4}$

A autoimagem do indivíduo é afetada e a forma como ele percebe e se relaciona com o seu corpo é modificada; fazendose necessário reelaborar a experiência e reformular a identidade para que a pessoa, assim reabilitada, prossiga com a sua vida de forma satisfatória e integrada. Neste sentido, Galvan \& Amiralian" pontuam que "a dificuldade em realizar a elaboração imaginativa desta perda pode tornar a amputação um acontecimento não integrado na vida de uma pessoa, com consequências prejudiciais à sua saúde e ao seu desenvolvimento".

Segundo Silva ${ }^{5}$ a imagem corporal é a representação mental do corpo existencial que vai se transformando a partir das experiências do indivíduo, tornando-se mais organizada e consciente. Para esta autora, "a alteração da imagem corpórea está fortemente ligada à autoestima pessoal, existindo uma correlação positiva entre a imagem corporal e a autoimagem, e entre a satisfação com o corpo e a autoestima global"5.
Desta forma, a imagem corporal está ligada intrinsecamente ao desenvolvimento e a qualidade de vida, representando o reflexo de desejos, emoções e das relações interpessoais. ${ }^{6}$

A Organização Mundial da Saúde (OMS) entende a qualidade de vida (QV) não apenas como a ausência de doença ou enfermidade, mas também como a percepção do indivíduo de sua posição na vida no contexto da cultura e sistema de valores, nos quais ele vive e em relação aos seus objetivos, expectativas, padrões e preocupações. ${ }^{7}$

Milioli et al. ${ }^{8}$ apontam as questões psicossociais como fatores relevantes para a QV das pessoas amputadas, pois a limitação funcional interfere na sua autonomia e independência alterando suas atividades e relações consigo e com o meio.

Conforme essas autoras: os fatores físicos para obter a QV são: condições do coto e clínica geral do paciente (comorbidades), características da prótese, tempo desde a amputação, capacidade de mobilidade com a prótese. Já, os fatores psicológicos valorizados e monitorados para a QV são: resiliência (mobilização pessoal para adaptar-se à nova realidade), aceitação da amputação, depressão (frequente em pacientes mais jovens ou que não vislumbram controle/manejo sobre sua deficiência) e otimismo. Por sua vez, incluemse como fatores psicossociais predisponentes à QV: participação em atividades sociais, trabalhar, estudar, convívio em grupo de amigos, associações, entre outros. ${ }^{8}$

Publicações relacionando amputação e à qualidade de vida e autoestima ainda são escassas. Em relação à qualidade de vida destacamos o artigo de Milioli et al. ${ }^{8}$ que avaliou 11 sujeitos submetidos a amputação, através do WHOQOL-Bref (World Health Organization Quality of Life) no qual $36,4 \%$ dos sujeitos avaliaram como nem ruim/nem boa sua qualidade de vida e $27,3 \%$, relataram como ruim e muito ruim.

Moro et al. ${ }^{9}$ realizaram estudo com 166 pacientes submetidos a amputação por etiologia vascular, que também responderam ao questionário WHOQOL-Bref, de acordo com esses autores, os pacientes apresentavam-se em uma faixa intermediária de QV e o domínio físico apresentou com maior porcentagem de fracasso e menor de sucesso.

Em relação à autoestima e amputação grande parte dos estudos referem-se à identidade e imagem pessoal e corporal. Reis et al. $^{10}$ fazem uma intervenção em terapia cognitivo-comportamental com 12 pacientes, visando reabilitar e melhorar a qualidade de vida dos mesmos, foram realizados 16 encontros semanais abordando assuntos como autoimagem, treino de resolução de problemas, dores fantasmas, entre outros. Os autores concluem que os indivíduos tornaram-se mais participativos e mais conscientes das dificuldades e proativos para a colocação da prótese.

Silva $^{5}$ realizou estudo experimental de cunho qualitativo e quantitativo, com 46 indivíduos buscando compreender o impacto da amputação na imagem corporal. $\mathrm{Na}$ avaliação, utilizou os instrumentos: BIS (Body Image Scale - escala de Imagem Corporal), o BSI, (Brief Symptom Inventory - Inventário de Sintomas Psicopatológicos), a ESSS (Escala de Satisfação com o Suporte Social), o QAEG (Questionário de Autoestima Global), e ainda um questionário sociodemográfico, clínico e psicossocial. Observou associações significativas entre a imagem corporal, a autoestima global, o suporte social, a depressão, a ansiedade e a idade.

Dentre as intervenções visando a melhor qualidade de vida, pontuamos a Arteterapia, que utilizando-se dos recursos expressivos e tendo como foco o indivíduo e suas necessidades, possibilita o paciente expressar-se espontaneamente e trazer um novo significado à vida. ${ }^{11}$ Desta forma, associada à reabilitação, pode favorecer a compreensão dos conteúdos emergentes advindos da amputação. E de acordo com Francisquetti ${ }^{12}$ "as atividades de ArteReabilitação favorecem a conscientização de um novo esquema corporal, promovendo uma nova estruturação, ajudando o indivíduo a suportar e transpor o sofrimento".

Publicações sobre Arteterapia e amputação, ainda são poucas no Brasil, destacamos dois estudos de caso que podem ser vistos, Montessanti \& Freire $^{13}$ e Alves. ${ }^{14}$ Essas autoras mostram o uso dos recursos artísticos, como a fotografia e a pintura como meios facilitadores para reestruturar a autoimagem e permitir a retomada da vida social. Destacam, também, a importância da equipe multidisciplinar e interdisciplinar integradas na reabilitação dos amputados.

A Arte-Reabilitação é importante aliada para auxiliar na reestruturação da imagem de um corpo que foi bruscamente modificado, além de propiciar melhora tanto da expectativa do processo de possível protetização quanto da motivação, autoestima e outros sentimentos decorrentes da lesão. ${ }^{13}$

Os contos e mitos são recursos que favorecem o desenvolvimento do trabalho arteterapêutico, Bernardo ${ }^{15}$ relata que 
"Um conto ou um mito, narrado ou utilizado como guia para um trabalho arteterapêutico no momento certo com a pessoa certa, tem o poder de abrir a escuta ao chamado da alma e suas necessidades atuais". Campbell ${ }^{16}$ ensina que os mitos podem nos ajudar no processo de autoconhecimento e evolução pessoal, pois fornecem "os símbolos que levam o espírito humano a avançar, opondo-se àquelas outras fantasias humanas constantes que tendem a levá-lo para trás."

As reações biopsicoemocionais advindas da amputação são fatores que contribuem para os distúrbios de comportamento e a baixa autoestima interferindo na reabilitação e a qualidade de vida. A Arteterapia utiliza-se dos recursos expressivos tendo como foco o indivíduo e suas necessidades buscando promover o autoconhecimento, o bem estar e a qualidade de vida. Devemos ser receptivos a todos os recursos que possam implementar o bem estar e a qualidade de vida dos pacientes amputados.

A hipótese deste estudo é que o atendimento arteterapêutico com enfoque no uso de história pode auxiliar no processo de reabilitação nos aspectos de qualidade de vida e autoestima em mulheres amputadas.

\section{OBJETIVO}

O objetivo deste estudo foi avaliar a influência de intervenção em Arteterapia na autoestima e qualidade de vida em mulheres amputadas.

\section{MÉTODO}

Foram contatadas 20 mulheres que se enquadravam nos critérios estabelecidos para a pesquisa, porém 8 aceitaram participar, os motivos para a não participação foram devido a indisponibilidade de horário e falta de interesse.
Sendo assim, participaram deste estudo 8 mulheres da clínica de amputados da Associação de Assistência a Criança Deficiente (AACD), com diagnóstico de amputação traumática e vascular, em idade de 35 a 65 anos, com ensino fundamental, fases protética e pré protética, em terapias e clinicamente estáveis, foram excluídas participantes que apresentassem déficit visual grave, déficit cognitivo, amputação de membros superiores e com diagnóstico de depressão.

Todas as participantes assinaram termo de consentimento livre e esclarecido e autorização de uso de imagem, e esta pesquisa foi aprovada pelo comitê de pesquisa e ética da instituição (CAAE 69612117.9.0000.0085).

Para a avaliação de início e fim de intervenção foram utilizados os seguintes instrumentos:

WHOQOL - BREF (World Health Organization Quality of Life) - constituído de 26 perguntas, sendo duas questões gerais e as demais representam 24 facetas as quais compõem quatro domínios que são: físico, psicológico, relações sociais e meio ambiente. As respostas seguem uma escala de Likert (de 1 a 5 , quanto maior a pontuação melhor a qualidade de vida).

Escala de Autoestima Rosenberg (EAR) - instrumento unidimensional capaz de classificar o nível de autoestima em baixo, médio e alto. Possui dez itens, sendo seis referentes a uma visão positiva de si mesmo e quatro referentes a uma visão autodepreciativa. As opções de resposta são "discordo plenamente", "discordo" "concordo", e "concordo plenamente". Em relação à pontuação, quanto maior o escore obtido na escala, maior o nível de autoestima do indivíduo.

Desenho autoimagem: Solicitado a cada participante "Desenhe você" em folha de A4 branca com caneta preta. Os aspectos formais do desenho são analisados de forma qualitativa: aspectos de tamanho e ocupação de espaço na folha, Gestalt, e uso de detalhes.
Segundo Francisquetti ${ }^{17}$ o desenho da figura humana é um reflexo do desenvolvimento individual e para a fenomenologia este esquema corporal expressa este estar no mundo.

As participantes (Controle e Intervenção) foram avaliadas por psicólogas da instituição. Os resultados não foram do conhecimento das pesquisadoras, a fim de evitar a contaminação na intervenção.

As participantes foram divididas em grupos: de intervenção (4 participantes) e Grupo Controle (4 participantes) constituindo uma amostra de conveniência (disponibilidade de participação e frequência na instituição).

Foram elaboradas oficinas temáticas baseada no mito "Caixa de Pandora". O mito de Pandora é um mito grego de Hesíodo, associado ao mito de Prometeu (aquele que pensa primeiro e depois age), que junto com seu irmão Epimeteu (aquele que age primeiro e depois pensa) criou os homens. Prometeu dá aos homens o fogo divino. Como castigo Zeus pede a seu filho Hefesto que crie uma linda mulher; e esta recebe o nome de Pandora (aquela que possui os dons divinos) é dada de presente a Epimeteu, com uma caixa misteriosa que em hipótese alguma deveria ser aberta. Em algumas versões do mito, Pandora, abre a caixa de onde saem todos os males do mundo, restando apenas a esperança no fundo; em outras versões, o próprio Epimeteu é quem abre a caixa. ${ }^{18,19}$

O grupo de intervenção participou de 11 oficinas arteterapêuticas temáticas (Tabela 1), estruturadas em sensibilização, atividade expressiva e fechamento, 1 vez por semana no setor de Arte Reabilitação da instituição.

A coleta de dados foi feita pelas pesquisadoras através do registro de imagens, gravação dos relatos e relatórios diários.

Tabela 1. Descrição das oficinas

\begin{tabular}{|c|c|c|c|}
\hline OF & TEMA & ATIVIDADE & OBJETIVOS \\
\hline 01 & Apresentação & Tela Coletiva & Formação de vínculo. \\
\hline 02 & A Notícia & Pintura em Tecido Molhado & Refletir sobre a nova possibilidade de vida com aceitação e acolhimento do novo corpo. \\
\hline 03 & Os Sentimentos & Modelagem em Argila & Perceber as reações emocionais. \\
\hline 04 & Os Dons e Talentos & Mandala dos Dons & Descobrir os potenciais e apoderar-se deles. \\
\hline 05 & Projetos Futuros & Garrafa Mágica & Planejar metas futuras. \\
\hline 06 & As Incertezas da Vida & Confecção da Caixa & Refletir sobre as incertezas da vida e sobre a curiosidade. \\
\hline 07 & Eu no Mundo & Confecção do Porta Retrato & Trabalhar o autoconhecimento, a autoaceitação e posicionar-se no mundo. \\
\hline 08 & Os Relacionamentos & Mandala da Fios & Refletir sobre as diferenças, a interferência dos outros, o dar e o receber, aceitar a ajuda. \\
\hline 09 & Os Males do Mundo e a Esperança & Transformação da Caixa & Refletir sobre a resiliência e a Esperança. \\
\hline 10 & A Esperança & Mandala da Velas & Manter a esperança acesa. Redescobrir o brilho interior. \\
\hline 11 & Fechamento & Ponte & Refletir sobre o processo arteterapêutico. \\
\hline
\end{tabular}


Os resultados do Whoqol-Bref e o EAR foram corrigidos e tabulados. A análise de dados foi composta por análises de correlação entre grupo controle e grupo de intervenção e análise de correlação de grupo de intervenção pré e pós-atividades. Os resultados dos dados foram analisados com o auxílio do programa SPSS V20 (Statistical Package for the Social Sciences),
Minitab 16 e Excel Office 2010. As análises estatísticasadotaramcomoníveldesignificância valores de $p<0,05$.

$O$ desenho de autoimagem foi analisado qualitativamente através de aspectos de tamanho e ocupação de espaço na folha, Gestalt, e uso de detalhes assim como os depoimentos das participantes a respeito das atividades expressivas.

Tabela 2. Comparação entre grupos Pré Intervenção para EAR

\begin{tabular}{lccccccc}
\hline EAR Inicial & & Média & Mediana & Desvio Padrão & N & IC & P-valor \\
\hline \multirow{2}{*}{ Autoconfiança } & Controle & 12,25 & 12,5 & 0,96 & 4 & 0,94 & 0,765 \\
& Intervenção & 12,50 & 12,5 & 1,73 & 4 & 1,70 & 0,94 \\
\multirow{2}{*}{ Autodepreciação } & Controle & 13,25 & 13,5 & 0,96 & 4 & 0,027 \\
& Intervenção & 11,00 & 11,0 & 0,82 & 4 & 0,80 & 0,57 \\
\multirow{2}{*}{ Total } & Controle & 25,50 & 25,5 & 0,58 & 4 & $1,0,063$ \\
\hline
\end{tabular}

Tabela 3. Comparação entre grupos Pré Intervenção para WHOQOL-Bref. Inicial

\begin{tabular}{|c|c|c|c|c|c|c|c|}
\hline WHOQOL-Bref. Inicial & Média & Mediana & Desvio Padrão & $\mathrm{N}$ & IC & P-valor & \\
\hline \multirow{2}{*}{ DOM.FISICO } & Controle & 69,64 & 69,6 & 8,50 & 4 & 8,33 & \multirow{2}{*}{0,297} \\
\hline & Intervenção & 63,39 & 62,5 & 3,42 & 4 & 3,35 & \\
\hline \multirow{2}{*}{ DOM.PSICO } & Controle & 81,25 & 81,3 & 12,95 & 4 & 12,70 & \multirow{2}{*}{0,191} \\
\hline & Intervenção & 67,71 & 64,6 & 14,97 & 4 & 14,68 & \\
\hline \multirow{2}{*}{ DOM.SOCIAL } & Controle & 68,75 & 70,8 & 20,83 & 4 & 20,42 & \multirow{2}{*}{0,462} \\
\hline & Intervenção & 60,42 & 62,5 & 14,23 & 4 & 13,95 & \\
\hline \multirow{2}{*}{ DOM.AMBIENTE } & Controle & 62,50 & 56,3 & 14,66 & 4 & 14,36 & \multirow{2}{*}{0,561} \\
\hline & Intervenção & 57,03 & 54,7 & 11,23 & 4 & 11,01 & \\
\hline \multirow{2}{*}{ QV } & Controle & 70,54 & 68,5 & 12,49 & 4 & 12,24 & \multirow{2}{*}{0,248} \\
\hline & Intervenção & 62,14 & 62,6 & 7,86 & 4 & 7,70 & \\
\hline
\end{tabular}

Tabela 4. Comparação entre grupos Pós Intervenção para EAR

\begin{tabular}{lcccccccc}
\hline Rosenberg Final & & Média & Mediana & Desvio Padrão & N & IC & P-valor \\
\hline \multirow{2}{*}{ Autoconfiança } & Controle & 11,25 & 11,5 & & 1,71 & 4 & 1,67 & 0,765 \\
& Intervenção & 11,75 & 11,5 & & 0,96 & 4 & 0,94 & \\
\multirow{2}{*}{ Autodepreciação } & Controle & 13,25 & 13,5 & 1,71 & 4 & 1,67 & 0,554 \\
& Intervenção & 12,50 & 13,0 & 1,91 & 4 & 1,88 & 1,70 & 1,000 \\
\multirow{2}{*}{ Total } & Controle & 24,50 & 24,5 & 1,73 & 4 & 1,70 \\
& Intervenção & 24,25 & 24,5 & 2,50 & 4 & 2,45 & \\
\hline
\end{tabular}

Tabela 5. Comparação entre grupos Pós intervenção Grupos para WHOQOL-Bref

\begin{tabular}{|c|c|c|c|c|c|c|c|}
\hline WHOQOL-Bref. Final & Média & Mediana & Desvio Padrão & $\mathrm{N}$ & IC & P-valor & \\
\hline \multirow{2}{*}{ DOM.FISICO } & Controle & 72,32 & 71,4 & 13,16 & 4 & 12,90 & \multirow{2}{*}{1,000} \\
\hline & Intervenção & 69,64 & 73,2 & 11,10 & 4 & 10,88 & \\
\hline \multirow{2}{*}{ DOM.PSICO } & Controle & 78,13 & 72,9 & 14,97 & 4 & 14,68 & \multirow{2}{*}{1,000} \\
\hline & Intervenção & 70,83 & 75,0 & 11,28 & 4 & 11,06 & \\
\hline \multirow{2}{*}{ DOM.SOCIAL } & Controle & 68,75 & 62,5 & 21,92 & 4 & 21,48 & \multirow{2}{*}{0,309} \\
\hline & Intervenção & 54,17 & 50,0 & 22,05 & 4 & 21,61 & \\
\hline \multirow{2}{*}{ DOM.AMBIENTE } & Controle & 65,63 & 60,9 & 18,58 & 4 & 18,20 & \multirow{2}{*}{1,000} \\
\hline & Intervenção & 63,28 & 68,8 & 13,10 & 4 & 12,84 & \\
\hline \multirow{2}{*}{ QV } & Controle & 71,21 & 64,8 & 16,26 & 4 & 15,93 & \multirow{2}{*}{0,663} \\
\hline & Intervenção & 64,48 & 64,5 & 11,09 & 4 & 10,86 & \\
\hline
\end{tabular}

\section{RESULTADOS}

Os grupos apresentaram-se estatisticamente homogêneos em relação à idade dos participantes, com média de idade de 46 anos para o grupo controle e 51 anos para intervenção.

Quanto à avaliação de pré-intervenção as participantes apresentam diferença significativa entre os grupos no EAR (Tabela 2), no domínio de autodepreciação e no total apresentam tendência a significância e não apresentaram diferenças significativas no Whoqol-Bref em préintervenção (Tabela 3).

Com relação aos resultados pós-intervenção, não foram apresentadas diferenças significativas entre grupos no EAR (Tabela 4) e no Whoqol-BREF (Tabela 5).

Quando comparados os resultados do EAR de início e fim dos grupos em seu desempenho, não foram verificadas diferenças significativas em ambos os grupos (Tabela 6 e 7). Porém verifica-se uma tendência a significância em autodepreciação para o grupo intervenção ( $p$-valor $=0,141)$.

Nos resultados do Whoqol-Bref quando comparados os desempenhos dos grupos de início e fim de intervenção, verificamos que não se apresentam diferenças significativas em ambos os grupos.

Com referência as análises dos desenhos de autoimagem, o grupo controle manteve o uso do espaço, não variou na Gestalt e uso de detalhes e houve de uma participante o menor tamanho da figura (Figura 1). No grupo de Intervenção verificou-se aumento do uso de detalhes, aumento do tamanho da figura, melhora na Gestalt e uso do espaço (Figura 2).

\section{DISCUSSÃO}

Este estudo teve por objetivo avaliar a influência da intervenção em Arteterapia na autoestima e qualidade de vida em mulheres amputadas.

Verificamos nos dados quantitativos apresentados que o grupo intervenção apresentava comparado ao grupo controle, tendência a uma menor autoestima no início da pesquisa (total $p$-valor $=0,063$ ) e uma diferença significativa no aspecto de autodepreciação medido pelo EAR ( $p$-valor $=0,027$ ), apontando para pacientes que tendiam a se depreciar em seu discurso. Este dado pode ser observado durante o início do processo, no discurso das participantes, como exemplo da participante $B$. quando relata ser difícil realizar a atividade e diz: "não consigo desenrolar eu mesma. O que eu imagino, eu não consigo transportar (para o papel)". 
Tabela 6. Comparação Inicial e Final EAR - Grupo Controle

\begin{tabular}{lccccccc}
\hline Rosenberg Controle & & Média & Mediana & Desvio Padrão & N & IC & P-valor \\
\hline \multirow{2}{*}{ Autoconfiança } & Inicial & 12,25 & 12,5 & 0,96 & 4 & 0,94 & 0,285 \\
& Final & 11,25 & 11,5 & 1,71 & 4 & 1,67 & 0,94 \\
\multirow{2}{*}{ Autodepreciação } & Inicial & 13,25 & 13,5 & 0,96 & 4 & 1,000 \\
& Final & 13,25 & 13,5 & 1,71 & 4 & 1,67 & 0,57 \\
\multirow{2}{*}{ Total } & Inicial & 25,50 & 25,5 & 0,58 & 4 & 1,73 & 0,285 \\
\hline
\end{tabular}

Tabela 7. Comparação Inicial e Final EAR - Grupo Intervenção

\begin{tabular}{|c|c|c|c|c|c|c|c|}
\hline \multicolumn{2}{|c|}{ Rosenberg Intervenção } & \multirow{2}{*}{$\frac{\text { Média }}{12,50}$} & \multirow{2}{*}{$\frac{\text { Mediana }}{12,5}$} & \multirow{2}{*}{$\frac{\text { Desvio Padrão }}{1,73}$} & \multirow{2}{*}{$\frac{N}{4}$} & \multirow{2}{*}{$\frac{\text { IC }}{1,70}$} & \multirow{3}{*}{$\frac{\text { P-valor }}{0,461}$} \\
\hline \multirow{2}{*}{ Autoconfiança } & Inicial & & & & & & \\
\hline & Final & 11,75 & 11,5 & 0,96 & 4 & 0,94 & \\
\hline \multirow{2}{*}{ Autodepreciação } & Inicial & 11,00 & 11,0 & 0,82 & 4 & 0,80 & \multirow{2}{*}{0,141} \\
\hline & Final & 12,50 & 13,0 & 1,91 & 4 & 1,88 & \\
\hline \multirow{2}{*}{ Total } & Inicial & 23,50 & 24,0 & 1,91 & 4 & 1,88 & \multirow{2}{*}{0,785} \\
\hline & Final & 24,25 & 24,5 & 2,50 & 4 & 2,45 & \\
\hline
\end{tabular}

Tabela 8. Comparação Inicial e Final Whoqol-Bref - Grupo Controle

\begin{tabular}{|c|c|c|c|c|c|c|c|}
\hline WHOQOL-Bref Controle & Média & Mediana & Desvio Padrão & $\mathrm{N}$ & IC & P-valor & \\
\hline \multirow{2}{*}{ DOM.FISICO } & Inicial & 69,64 & 69,6 & 8,50 & 4 & 8,33 & \multirow{2}{*}{0,715} \\
\hline & Final & 72,32 & 71,4 & 13,16 & 4 & 12,90 & \\
\hline \multirow{2}{*}{ DOM.PSICO } & Inicial & 81,25 & 81,3 & 12,95 & 4 & 12,70 & \multirow{2}{*}{0,655} \\
\hline & Final & 78,13 & 72,9 & 14,97 & 4 & 14,68 & \\
\hline \multirow{2}{*}{ DOM.SOCIAL } & Inicial & 68,75 & 70,8 & 20,83 & 4 & 20,42 & \multirow{2}{*}{0,854} \\
\hline & Final & 68,75 & 62,5 & 21,92 & 4 & 21,48 & \\
\hline \multirow{2}{*}{ DOM.AMBIENTE } & Inicial & 62,50 & 56,3 & 14,66 & 4 & 14,36 & \multirow{2}{*}{0,465} \\
\hline & Final & 65,63 & 60,9 & 18,58 & 4 & 18,20 & \\
\hline \multirow{2}{*}{ QV } & Inicial & 70,54 & 68,5 & 12,49 & 4 & 12,24 & \multirow{2}{*}{1,000} \\
\hline & Final & 71,21 & 64,8 & 16,26 & 4 & 15,93 & \\
\hline
\end{tabular}

Tabela 9. Comparação Inicial e Final Whoqol-Bref - Grupo Intervenção

\begin{tabular}{|c|c|c|c|c|c|c|c|}
\hline WHOQOL-Bref Intervenção & Média & Mediana & Desvio Padrão & $\mathrm{N}$ & IC & P-valor & \\
\hline \multirow{2}{*}{ DOM.FISICO } & Inicial & 63,39 & 62,5 & 3,42 & 4 & 3,35 & \multirow{2}{*}{0,197} \\
\hline & Final & 69,64 & 73,2 & 11,10 & 4 & 10,88 & \\
\hline \multirow{2}{*}{ DOM.PSICO } & Inicial & 67,71 & 64,6 & 14,97 & 4 & 14,68 & \multirow{2}{*}{0,593} \\
\hline & Final & 70,83 & 75,0 & 11,28 & 4 & 11,06 & \\
\hline \multirow{2}{*}{ DOM.SOCIAL } & Inicial & 60,42 & 62,5 & 14,23 & 4 & 13,95 & \multirow{2}{*}{0,285} \\
\hline & Final & 54,17 & 50,0 & 22,05 & 4 & 21,61 & \\
\hline \multirow{2}{*}{ DOM.AMBIENTE } & Inicial & 57,03 & 54,7 & 11,23 & 4 & 11,01 & \multirow{2}{*}{0,465} \\
\hline & Final & 63,28 & 68,8 & 13,10 & 4 & 12,84 & \\
\hline \multirow{2}{*}{ QV } & Inicial & 62,14 & 62,6 & 7,86 & 4 & 7,70 & \multirow{2}{*}{0,465} \\
\hline & Final & 64,48 & 64,5 & 11,09 & 4 & 10,86 & \\
\hline
\end{tabular}

Ao final das intervenções não foi verificado diferença entre os grupos, porém o discurso da paciente modifica quando relata "Gostei muito da terapia, senti que ajudou muito na autoestima, senti que sou capaz, aprendi que tem que ter perseverança e que tudo na vida é surpresa. Surpresa às vezes boa, para melhor. Gostei de todas as etapas do trabalho em conjunto. Foram todos importantes para mim".
Este discurso corrobora com a fala de Andrade et al. ${ }^{20}$ sobre o conceito de autoestima, quando relata que esta faz parte do autoconceito, e que o indivíduo expressa sentimentos ou atitude de aprovação e repulsa por si mesmo. O sujeito será capaz de considerar-se capaz, bem sucedido e valioso.

Os dados quantitativos referentes à qualidade de vida (Whoqol-Bref) e Autoestima
(EAR), não apresentaram diferenças estatísticas, levantamos a hipótese que o número de participantes não foi suficiente para apontar melhora dos dois aspectos avaliados através dos instrumentos aplicados.

A abordagem qualitativa da análise do desenho e do discurso das participantes possibilitou um olhar mais sensível e amplo à questão da autoestima e qualidade de vida, captando mudanças que não foram identificadas pelos instrumentos quantitativos.

Machover apud Silva et al. ${ }^{21}$ afirma: "quando um sujeito realiza um desenho está se referindo às imagens internalizadas que tem de si e dos outros. O desenho, assim, representa a expressão do eu no ambiente". Essas autoras concluem que:

A linguagem gráfica é a está mais próxima do inconsciente e do ego corporal; (...) seu conteúdo sofre menor influência do consciente (...). No caso de pacientes com dificuldades em se expressar verbalmente, é uma excelente ferramenta para facilitar a comunicação. $^{21}$

Em relação a análise do desenho, verificamos que o grupo de intervenção tendeu a apresentar um desenho ao final com aumento de detalhes. A ausência de detalhes pode indicar uma tendência à introspecção e retraimento ${ }^{22,23}$ no discurso foi possível verificar que a fala das participantes apontam para a percepção de suas qualidades e possibilidades como: "percebi que tenho que fazer o meu melhor"; " a gente é capaz", "a força, o brilho, a fé e a esperança estão sempre presentes. Para brilhar é preciso ser você mesmo".

Também foi possível observar um aumento no tamanho da figura humana e aumento no uso do espaço, que segundo Francisquetti ${ }^{22}$ apontam para um comportamento mais expansivo e de autovalorização e Levy ${ }^{24}$ pontua que o espaço pode apresentar um paralelo entre o sujeito e seu ambiente, figuras grandes podem denotar como o sujeito está reagindo as pressões ambientais e figuras pequenas podem ser vistas como o sujeito sentir-se pequeno (inferior) ou perdido (rejeitado). Em um paralelo com o discurso das participantes notamos essa expansão em falas como: "tenho mais ânimo na minha vida"; "preciso experimentar os meus limites"; "quero encontrar uma pessoa para amar"; "a capacidade que eu tenho é muito grande, mas às vezes, a gente se esquece disso".

A Gestalt, segundo Francisquetti ${ }^{22}$ denota a ampliação da percepção de si e do meio, e pode ser observada sua melhora nos desenhos finais das participantes do grupo intervenção, 
em suas falas, as mesmas relatam situações como: "me deu um despertar"; "antes eu só vestia preto, agora uso vermelho e o vermelho fica bom em mim"; "abri minha mente para os meus projetos futuros"; "eu estou bem no mundo"; "estou toda colorida e cheia de flores".

A alteração do desenho das participantes corrobora com Silva ${ }^{5}$ quando a autora relata que a alteração da imagem corpórea está fortemente ligada à autoestima pessoal, existindo uma correlação positiva entre a imagem corporal e a autoimagem, e entre a satisfação com o corpo e a autoestima global. Este fato pode ser observado através do desenho por se tratar de uma linguagem gráfica mais próxima ao inconsciente, conforme pontua Silva et al. ${ }^{21}$ Esta atitude da modificação do desenho contribui para a afirmação de Montessanti e Freire $^{13}$ quando relatam que a Arte-Reabilitação poderá apresentar-se como aliada no trabalho da reestruturação da imagem corporal e também expectativa do processo de protetização, além do trabalho estimulando autoestima e outros sentimentos após a lesão.

De acordo com o processo terapêutico podemos observar que as participantes se mostram com mais identidade e completude, aceitando-se melhor e mais capazes de serem inseridas em outros contextos de suas vidas, como o relato da participante, que diz: "eu sou inteligente, vou dar um jeito nesta minha vida de deficiente e de profissional" (Figura 3).

Poucos são ainda os estudos que utilizam a associação dos instrumentos utilizados neste estudo (Whoqol e EAR) aliados à um processo de intervenção. Destacamos a pesquisa de Andrade et al. ${ }^{22}$ que fazem uma intervenção utilizando-se destes instrumentos visando a autoestima e qualidade de vida dos policiais civis do Rio de Janeiro, a qual resultou em acréscimos na qualidade de vida e cognição, de forma estatisticamente significativa nos domínios "recreação", "intimidade" e na "memória imediata". Com base nos ganhos significativos, os autores sugerem que tal prática pode ser válida para melhorar a condições de saúde de idosos institucionalizados.

Não foram encontrados na literatura nenhuma pesquisa que associe a Arteterapia e a amputação, sendo verificado através de instrumentos para avaliar a qualidade de vida e autoestima.

Daí apontamos a relevância deste um estudo no sentido de configurar a Arteterapia uma atuação importante no processo de reabilitação. Sugerimos, desta forma, a continuidade e ampliação deste estudo, atendendo as necessidades de sensibilidade

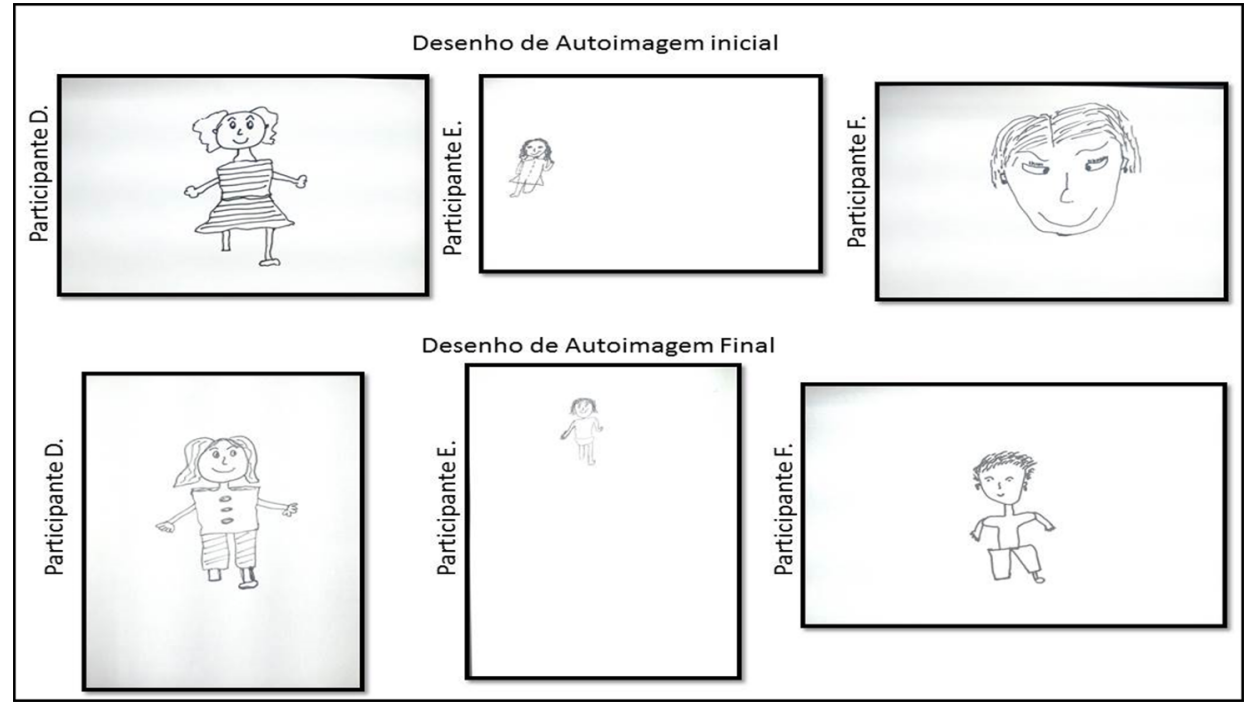

Figura 1. Desenho Início e Fim do Grupo de Controle

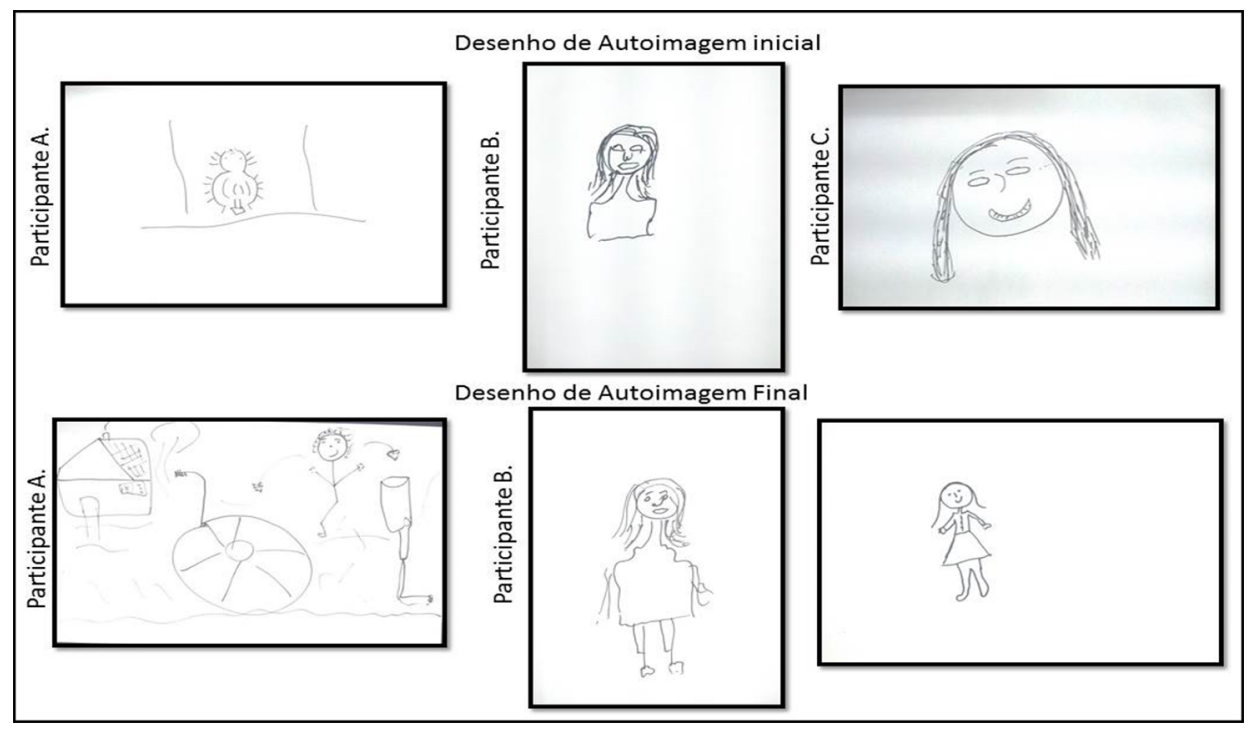

Figura 2. Desenho Início e Fim do Grupo de Intervenção

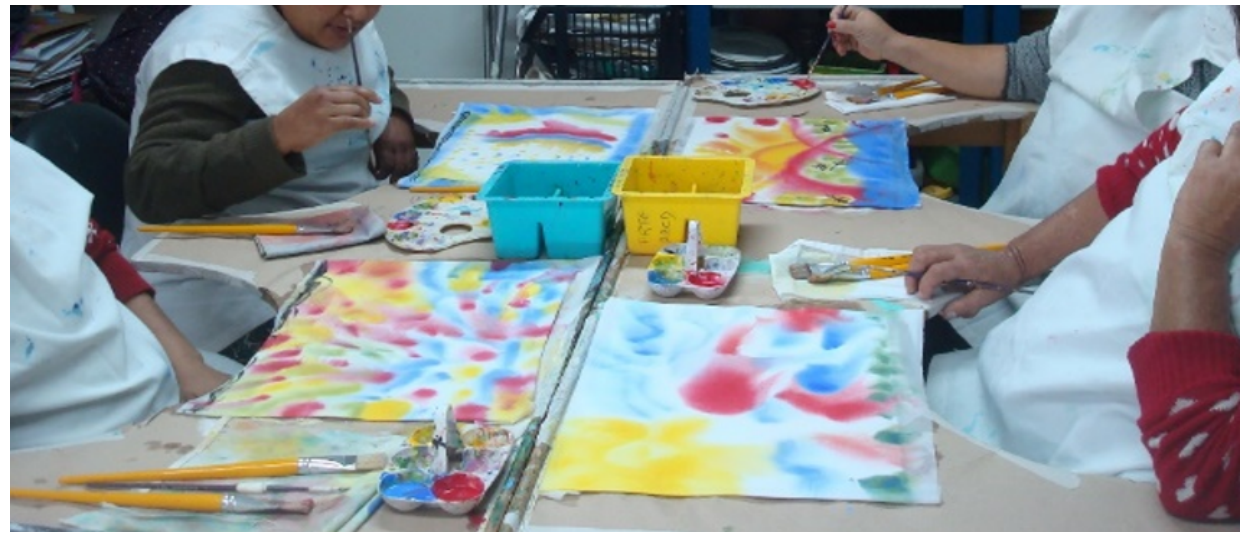

Figura 3. Pintura em tecido molhado 
ao teste para que os resultados possam também ser comprovados quantitativamente. Tomando como exemplo as estatísticas, em especial o desvio padrão e uma diferença nominal de 1,17 (IC de 95\%), do Rosenberg Inicial, temos que com um poder de $80 \%$ a amostra deveria ser de 17 sujeitos.

As oficinas de Arteterapia possibilitaram às participantes uma "pausa" na rotina diária da reabilitação, para que elas pudessem: olhar para si, refletir e se perceber (Figura 4).

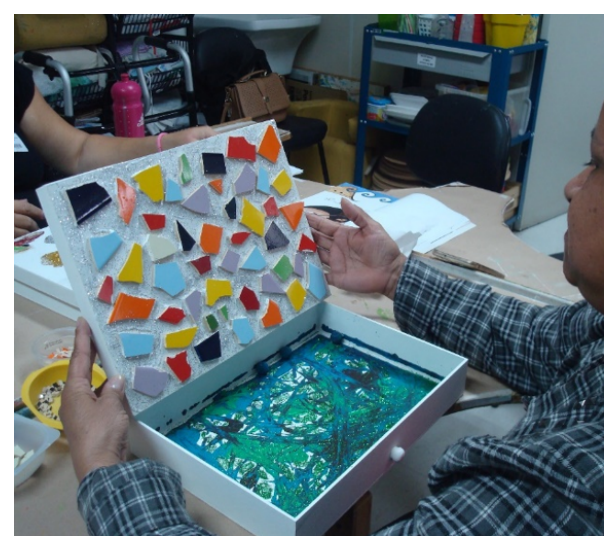

Figura 4. Transformação da caixa

Arteterapia pode contribuir para o processo de reabilitação do paciente, junto à equipe interdisciplinar pois, reabilitar não é apenas devolver as condições físicas necessárias ao paciente para que este possa se reinserir socialmente, a reabilitação deve ser capaz de fazer o paciente vivenciar um existir modificado, permitindo-se novas perspectivas e abertura à vida, mesmo que com novos redimensionamentos. Pois, a Arteterapia considera todas as experiências vividas pelos pacientes valiosas num sentido de tomada de consciência da sua singularidade e potenciais, ampliando o olhar deste indivíduo, atenuando as incapacidades provocadas pela amputação e permitindo um tratamento integral. Colaborando, consequentemente, com a promoção da autoestima e a qualidade de vida dessas pessoas.

0 arte-reabilitador deve ser sensível ao impacto do comprometimento físico na estrutura emocional e na autoestima dos pacientes buscando estabelecer com eles um vínculo amoroso e acolhedor e estimulando-os a perceber, expressar e elaborar suas emoções. Até porque sabemos que reabilitação se processa melhor quando o paciente está confiante e de bem com a vida. Em especial, cabe ao arteterapeuta que trabalha com reabilitação ajudar o paciente a perceber que apesar de seus comprometimentos, continua ser uma pessoa que pode produzir e criar- o que é de extrema valia em momentos em que o paciente, por sua condição, se sente deprimido ou inferiorizado. ${ }^{22}$

Desta forma, a Arteterapia impulsiona a pessoa a dar um passo adiante na fronteira onde o seu crescimento precisa acontecer, expandindo a criatividade e a liberdade de agir com mais consciência e eficiência aumentando desta forma, o seu repertório de comportamentos frente às várias situações de vida (Figura 5).

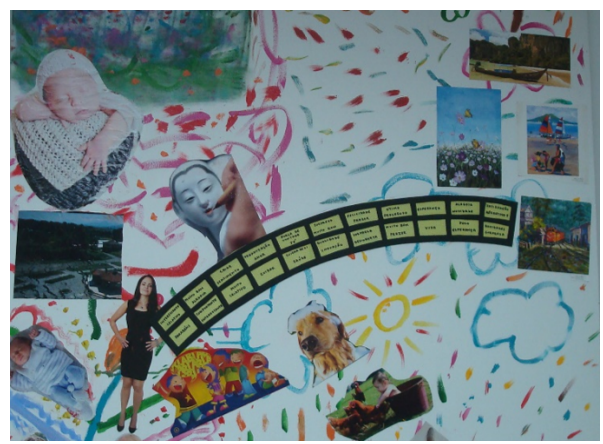

Figura 5. Trabalho de Construção da Ponte

\section{CONCLUSÃO}

O processo vivido por essas participantes foi repleto de símbolos e aprofundamento não apenas do processo de reabilitação, mas a continuidade de vida após a amputação. O trabalho desenvolvido através dos 11 encontros demonstrou a importância do processo arteterapêutico, através das atividades realizadas e as falas registradas, discutir as questões emocionais advindas do processo de amputação, e também trazer a luz a importância de carregar dentro da nossa "caixa" contendo os males do mundo, a esperança que permanece em cada pessoa, para seguir apesar das adversidades.

\section{REFERÊNCIAS}

1. Ramos ACR, Ingham SJM, Rolim Filho EF. Amputações. In: Fernandes AC, Ramos ACR, Morais Filho MC, Ares MJJ. Reabilitação. 2 ed. Barueri: Manole; 2014. p. 227-46.

2. Chini GCO, Boemer MR. A amputação na percepção de quem a vivencia: um estudo sob a ótica fenomenológica. Rev Latino-Am Enfermagem. 2007; 15(2):330-6.
3. Marques MSQ. Sentimento de perda: vivência da mulher com amputação de membro inferior [Dissertação] Porto: Universidade do Porto; 2008.

4. Galván GB, Amiralian MLTM. Corpo e identidade: reflexões acerca da vivência de amputação. Estud psicol. 2009;26(3):391-8

5. Silva MS. A imagem corporal na amputação: relação com a depressão, a ansiedade, a satisfação com o suporte social e a autoestima global [Dissertação]. Porto: Universidade do Porto; 2013.

6. Schilder P. A imagem do corpo: as energias construtivas da psique. São Paulo: Martins Fontes; 1999.

7. Fleck MPA. O instrumento de avaliação de qualidade de vida da Organização Mundial da Saúde (WHOQOL-100): características e perspectivas. Ciênc Saúde Coletiva. 2000;5(1):33-8.

8. Milioli R, Vargas MAO, Leal SMC, Montiel AA Qualidade de vida em pacientes submetidos à amputação. Rev Enferm UFSM. 2012;2(2):311-9.

9. Moro A, Assef MG, Araújo WS. Avaliação da qualidade de vida em pacientes submetidos à amputação de membros inferiores. Arq Catarin Med. 2012;41(1): 41-6.

10. Reis HR, Schuwab JA, Neufeld CB. Relato de experiência de Terapia Cognitivo-Comportamenta em Grupo com pacientes amputados. Rev Bras Ter Comp Cogn. 2014; XVI(2):148-64.

11. Sei MB. Arteterapia e psicanálise. São Paulo: Zagodoni; 2011.

12. Francisquetti AA. Arte-reabilitação: um caminho inovador na área da arteterapia. In: Francisquetti AA. Arte-Reabilitação. Rio de Janeiro: Wak; 2016.

13. Montessanti L, Freire TC. A arte-habilitação ou reabilitação? A descoberta de um novo caminho. In: Francisquetti AA. Arte-reabilitação. São Paulo: Memnon; 2011. p.18-25.

14. Alves CF. Amputação: um convite ao olhar e ao tocar as feridas do corpo e da alma. In: Francisquetti AA. ArteReabilitação. Rio de Janeiro: Wak; 2016. p.169-82.

15. Bernardo PP. Mitologia africana e arteterapia: a força dos elementos em nossa vida. São Paulo: Ed. Autor; 2009.

16. Campbell J. O herói de mil faces. 11 ed. São Paulo: Pensamento; 2007.

17. Francisquetti AA. A arte em um centro de reabilitação. Psicol Cienc Prof. 1992;12(1):34-8.

18. Brandão JS. Mitologia Grega. Petrópolis: Vozes; 1986.

19. Salis VD. Mitologia viva - aprendendo com os deuses a arte de viver e amar. São Paulo: Nova Alexandria; 2003.

20. Andrade ER, Souza ER, Minayo MCS. Intervenção visando a auto-estima e qualidade de vida dos policiais civis do Rio de Janeiro. Ciênc Saúde Coletiva. 2009;14 (1):275-85

21. Silva RBF, Pasa A, Castoldi DR, Spessatto F. O desenho da figura humana e seu uso na avaliação psicológica. Psicol Argum. 2010;28(60):55-64.

22. Francisquetti AA. Arte-reabilitação com portadores de paralisia cerebral. In: Ciornai S. Percursos em arteterapia: arteterapia gestaltica, arte em psicoterapia, supervisão em arteterapia. São Paulo: Summus; 2005. p. 239-50.

23. Hammer EF. Aspectos expressivos dos desenhos projetivos. In: Hammer EF. Aplicação clínica dos desenhos projetivos. São Paulo: Casa do Psicólogo; 1991. p. 42-60.

24. Levy S. Desenhos projetivos da figura humana. In: Hammer EF. Aplicação clínica dos desenhos projetivos. São Paulo: Casa do Psicólogo; 1991. p. 61-85. 